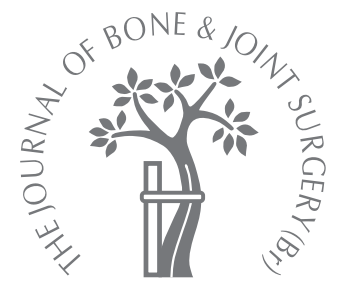
A. Eren,
H. Ömeroglu,
M. Güven,
E. Ugutmen,
F. Altintas

From the SB Göztepe Teaching and

Research Hospital, Istanbul and The

Osmangazi

University Hospital, Eskisehir, Turkey

\footnotetext{
A. Eren, MD, Orthopaedic Surgeon, Vice-Chief of the Clinic

M. Güven, MD Orthopaedic Surgeon

E. Ugutmen, MD,

Orthopaedic Surgeon

F. Altintas, MD, Associate

Professor, Chief of the Clinic

2nd Clinic of Orthopaedics

and Traumatology

SB Göztepe Teaching and

Research Hospital, Istanbul,

Turkey.

H. Ömeroglu, MD,

Professor

Department of Orthopaedics and Traumatology

Osmangazi University,

Faculty of Medicine, 26480

Eskisehir, Turkey.

Correspondence should be sent to $\mathrm{H}$. Ömeroglu at

Bitistiren Sokak 30/2, 06570

Ankara, Turkey; e-mail:

homeroglu@

superonline.com
}

(C)2005 British Editorial

Society of Bone and

Joint Surgery

doi:10.1302/0301-620X.87B6.

$15318 \$ 2.00$

$J$ Bone Joint Surg $[\mathrm{Br}]$

2005;87-B:790-5.

Received 19 January 2004;

Accepted after revision

1 October 2004

\title{
Incomplete triple pelvic osteotomy for the surgical treatment of dysplasia of the hip in adolescents and adults
}

\begin{abstract}
We treated 26 hips (24 consecutive patients) with residual dysplasia by a technique of incomplete triple pelvic osteotomy. The mean age of the patients was 21.6 years.

The mean values for the pre-operative centre-edge angle of Wiberg, the refined centreedge angle, the acetabular angle of Sharp, the modified acetabular angle and femoral head lateralisation were $7.7^{\circ},-3.1^{\circ}, 49.3^{\circ}, 53.2^{\circ}$ and $17.2 \mathrm{~mm}$, respectively. After a mean follow-up of 3.3 years they were $27.0^{\circ}, 13.0^{\circ}, 38.9^{\circ}, 44.3^{\circ}$ and $15.9 \mathrm{~mm}$, respectively $(p<0.05)$. The osteoarthritic grading changed adversely in one hip. The mean pre-operative and latest Harris hip scores were 74.9 and 93.0, respectively $(p<0.05)$.

This technique provides a stable osteotomy with maintenance of the posterior column which allows early mobilisation and minimal internal fixation. The technique is not complex and requires minimal blood transfusion. The use of an image intensifier is not necessary and harvesting of a subcristal bone graft avoids post-operative complications at the donor site.
\end{abstract}

Developmental dysplasia of the hip (DDH) may cause symptoms in later life. Surgical treatment by redirectional pelvic osteotomy is often recommended in adolescents and young adults. ${ }^{1}$ A technique of triple pelvic osteotomy was first described by LeCoeur, ${ }^{2}$ nearly 40 years ago, and since then several types of acetabular redirectional osteotomy including single, double and triple pelvic osteotomies and periacetabular and rotational acetabular procedures have been described..$^{3-6}$

In this prospective study, we have evaluated a modification of triple pelvic osteotomy including an incomplete curvilinear iliac osteotomy.

\section{Patients and Methods}

Between 1995 and 2001, 26 hips in 24 consecutive patients with residual hip dysplasia due to $\mathrm{DDH}$ were treated by an incomplete triple pelvic osteotomy by one of the authors (AE). Neuromuscular and teratological conditions were excluded. The mean age at operation was 21.6 years (11 to 41 ) and 19 patients were female. The right side was affected in 13 patients, the left in nine and both in two. Closed reduction and casting had been performed previously in six hips and combined pelvic and proximal femoral osteotomy in one. The remaining 19 hips had had no previous treatment. Two patients with bilateral involve- ment were operated on in two separate sessions one year apart.

The main complaint of all the patients was pain in the hip for at least six months. A hip was considered to be dysplastic if the centreedge (CE) angle of Wiberg ${ }^{7}$ was less than $20^{\circ}$ and the acetabular angle of Sharp ${ }^{8}$ greater than $43^{\circ 9}$ on a standard anteroposterior radiograph. The redirectional osteotomy was advised if the congruency and containment of the hip were considered to be acceptable on an anteroposterior pelvic radiograph with the proximal femur abducted and internally rotated. ${ }^{1}$ Routine preoperative or post-operative CT or MRI was not performed. Poor congruency, an open triradiate cartilage, complete dislocation of the hip and advanced osteoarthritis (Tönnis grade $3^{9}$ ) were contraindications.

Operative technique. An image intensifier is not required and the operation is performed through two separate incisions. The first step is an ischial osteotomy. The patient is placed supine with the affected hip elevated by $45^{\circ}$ by a pillow placed under the buttock. The hip and knee are slightly flexed by an assistant and an incision $6 \mathrm{~cm}$ long is made parallel to and $1 \mathrm{~cm}$ superior to the gluteal crest. The ischial ramus is exposed subperiosteally after blunt dissection through gluteus maximus. Two retractors are placed in the obturator foramen and the ischial osteotomy made just proximal to the 


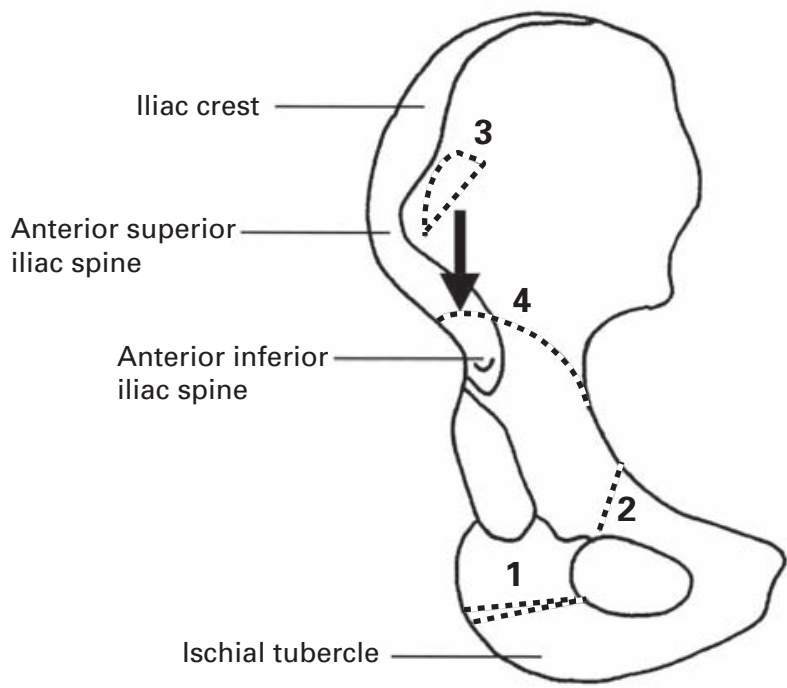

Fig. 1a

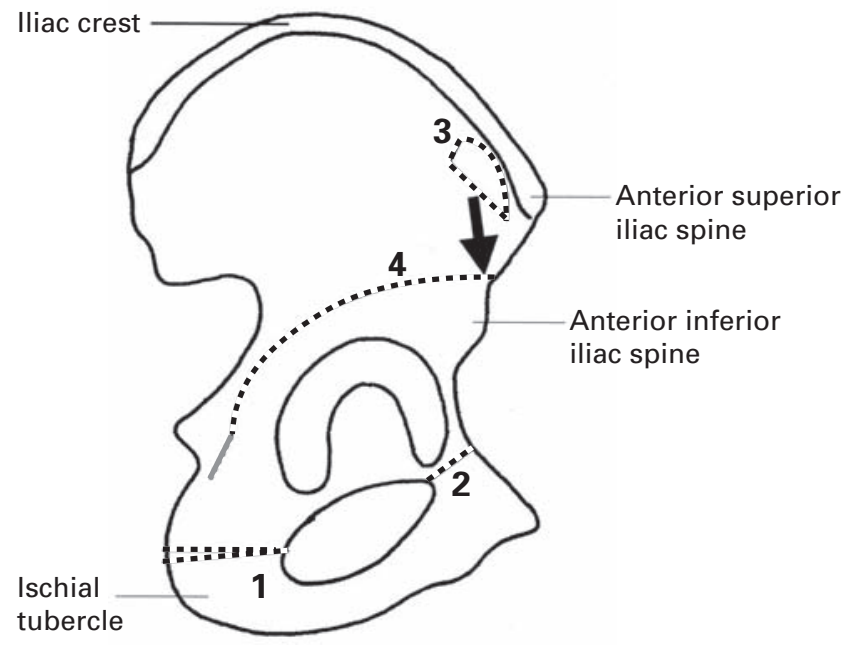

Fig. 1b

Diagram showing a) anteroposterior and b) lateral views of the surgical technique. 1, ischial osteotomy; 2, pubic osteotomy; 3 , subcristal bone graft; and 4, iliac osteotomy. The grey line in Figure $1 \mathrm{~b}$ represents the greenstick fracture produced after performing the iliac osteotomy.

origin of biceps femoris. A bone wedge, 7 to $8 \mathrm{~mm}$ wide based posterolaterally, is resected to facilitate later acetabular redirection (Fig. 1). The wound is closed without drainage.

The second part of the operation involves pubic and iliac osteotomies with acetabular redirection. The pillow is removed and an ilioinguinal incision $15 \mathrm{~cm}$ long is made along the anterior third of the iliac crest then on towards the pubic ramus. The lateral femoral cutaneous nerve is protected. Gluteus medius and minimus are sharply dissected and the lateral aspect of the iliac wing exposed subperiosteally. The abdominal and iliac muscles are not detached from the medial aspect of the iliac wing, but the medial and lateral aspects of the bony areas between the anterior superior (ASIS) and inferior (AIIS) iliac spines are exposed subperiosteally and two retractors placed into the sciatic notch. Tensor fascia lata is detached from the ASIS and the sartorius retracted medially. Rectus femoris is not detached. On the deep surface of iliopsoas the tendon is exposed and divided. The pubic ramus is exposed subperiosteally and two retractors placed under the ramus. The pubic osteotomy is $1 \mathrm{~cm}$ medial to the iliopubic eminence (Figs 1 and 2). A subcristal triangular curved bone graft is taken 1 to $2 \mathrm{~cm}$ posterior to the ASIS from the lateral aspect of the iliac wing using an oscillating saw. The size of the graft is related to the amount of correction and is usually about $4 \times 4 \times 2 \mathrm{~cm}$ (Figs 1, 2a and 2c). The iliac osteotomy is started $2 \mathrm{~cm}$ superior to the ASIS and is similar to that in a previously described combined pelvic osteotomy. ${ }^{10} \mathrm{~A}$ curvilinear cut is made parallel to the dome of the acetabulum proceeding posteriorly $1 \mathrm{~cm}$ above the sciatic notch into the body of the ischium where it terminates (Fig. 1). The first third of the osteotomy is performed using an oscil- lating saw followed by a curved sharp osteotome. Entering the acetabulum or sciatic notch should be avoided. It is possible to complete all of the medial and two-thirds of the lateral sides of the iliac osteotomy under direct vision. However, one-third of the distal part of the lateral side of the osteotomy is directed posterior to the joint capsule and into the body of the ischium without direct visualisation. The osteotomy is completed by producing a greenstick fracture in the remaining portion of the ischial body by opening the site of the iliac osteotomy and putting the limb in the 'figure-of-four' position (abduction and external rotation of the hip and flexion of the knee). The acetabulum is redirected to the desired position and the bone graft wedged into the osteotomy to maintain this (Fig. 3). A 2-mm Kirschner wire is used to fix the bone graft. After testing the movement of the hip and taking a radiograph, the wound is closed without drainage. Immobilisation of the hip is unnecessary. Partial weight-bearing $(10 \mathrm{~kg})$ with crutches and active movements of the hip are started on the third post-operative day. Full weight-bearing with crutches is allowed after radiological verification of bony healing, which usually occurs six to eight weeks after operation.

Radiological and clinical assessment. All the pre-operative and the latest follow-up radiographs were assessed by one of the authors ( $\mathrm{HO})$ who was blinded to the clinical status of the patients. The most lateral point of the bony acetabular roof and of the subchondral sclerosis (sourcil) in an anteroposterior radiograph reflected the most lateral point of the anterior and middle/posterior portions of the acetabulum in the transverse plane, respectively. ${ }^{11,12}$ The CE angle of Wiberg ${ }^{7}$ and the refined CE angle ${ }^{12}$ were used to determine the cover of the anterior and middle/posterior areas of the femoral head. The acetabular angle of Sharp ${ }^{8}$ and the 

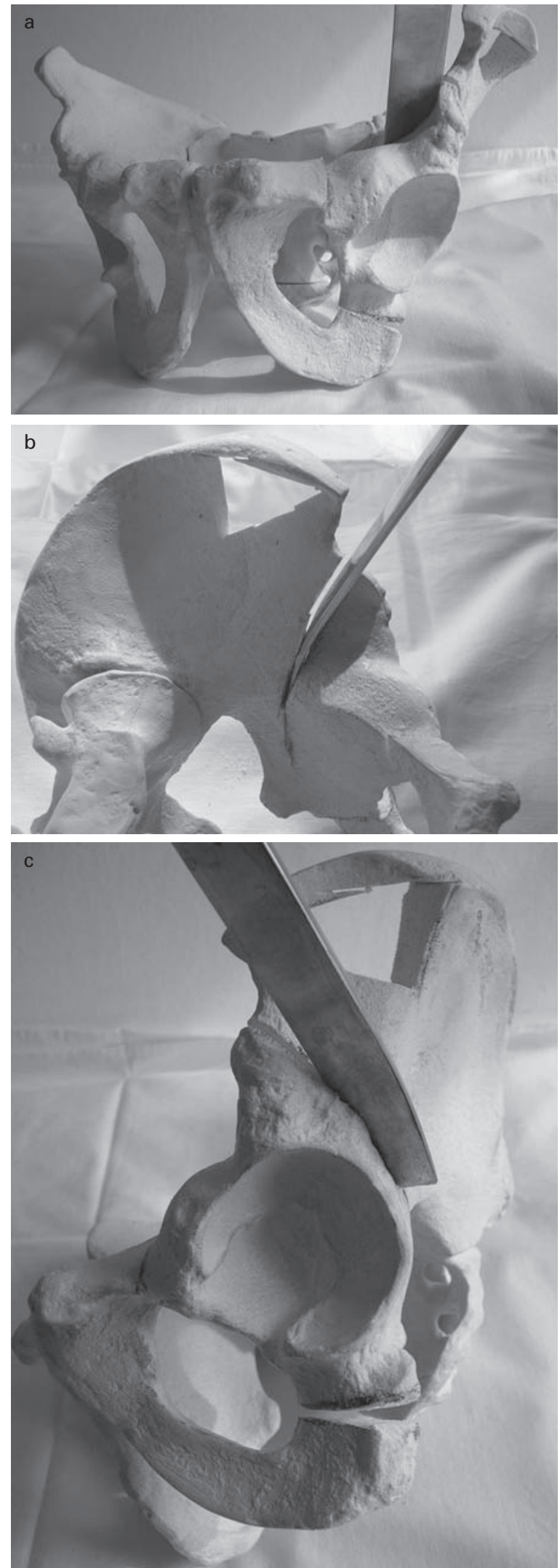

Fig. 2

Photographs of the technique in a model of a pelvis showing a) anteroposterior, b) medial and c) lateral views of the left pelvis during iliac osteotomy after completion of the ischial and pubic osteotomies and harvesting of the subcristal bone graft.

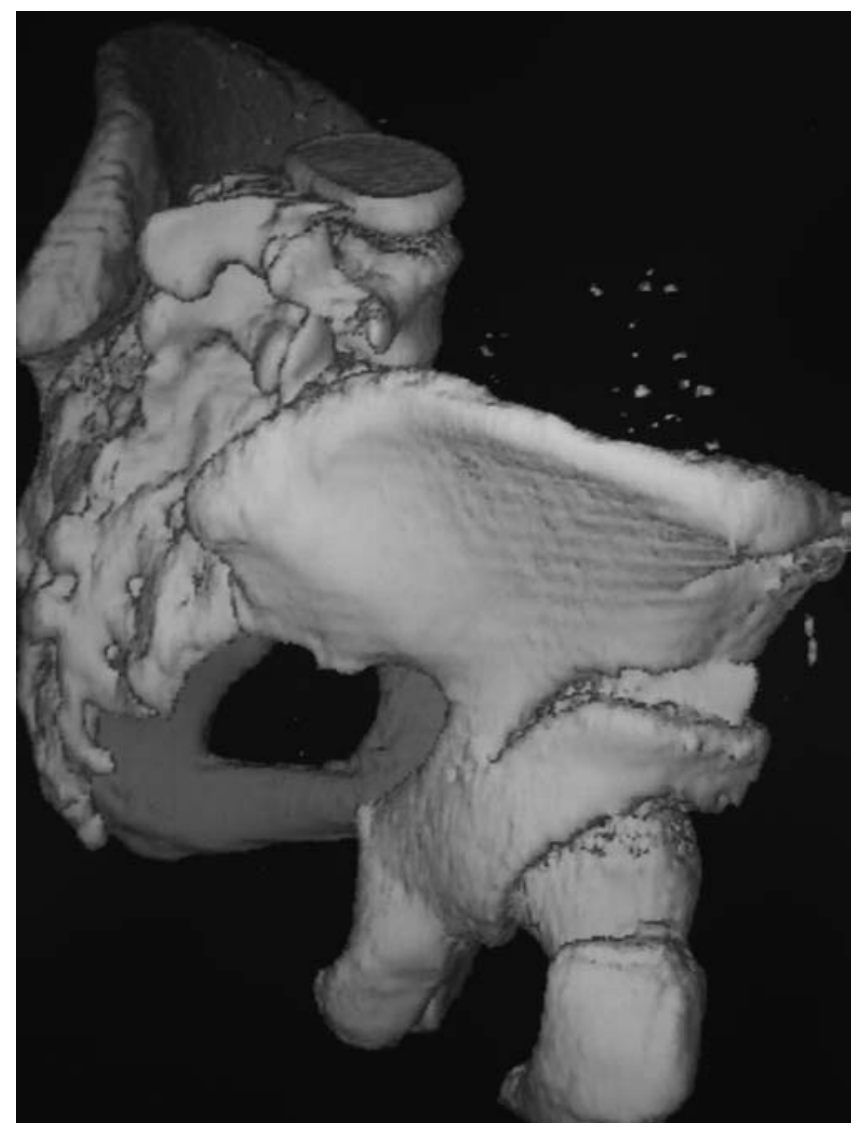

Fig. 3

Post-operative three dimensional CT scan of the right hip of a 30-year-old woman showing acetabular redirection after the triangular bone graft has been wedged into the iliac osteotomy.

modified acetabular angle ${ }^{13}$ were used to determine the slopes of the anterior and middle/posterior portions of the acetabulum. In the modified technique the most lateral point of the sourcil was used as the landmark. ${ }^{12,13}$ Since all these methods are reported to have adequate levels of intraand inter-observer reliability, ${ }^{13,14}$ an additional study was not done for this purpose. Lateralisation of the femoral head was measured as the distance in millimetres between the medial edge of the head and the ilioischial line. ${ }^{15}$ Osteoarthritic changes were graded according to Tönnis. ${ }^{9}$

Clinical assessment of the hips using the Harris hip score, ${ }^{16}$ was carried out by two of the authors (MG, EU) who were not primarily involved in the operations.

Statistical analysis. The $t$-test for paired samples compared the pre- and post-operative radiological parameters and the Wilcoxon signed-rank test was used for the comparison of the clinical scores. A p value of $<0.05$ was considered to be significant.

\section{Results}

Radiological and clinical. The operating time was between 1.5 and 2.5 hours and the blood loss less than $400 \mathrm{ml}$ in all 


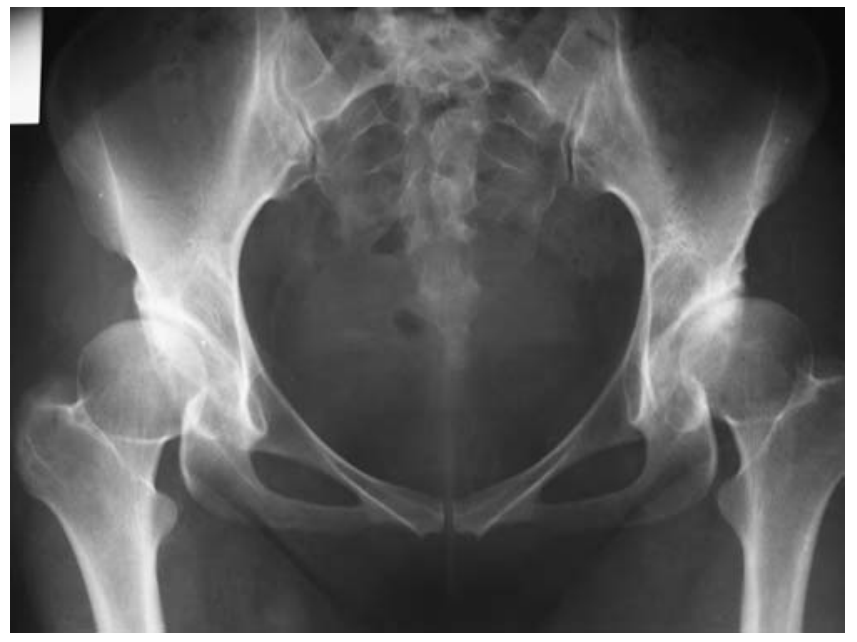

Fig. 4a

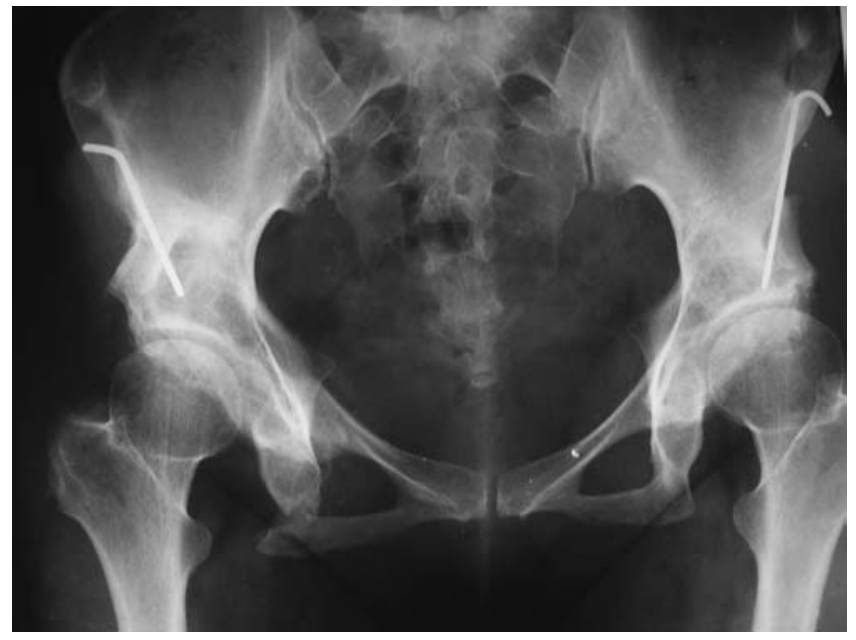

Fig. 4b

Anteroposterior pelvic radiographs of a woman with bilateral residual hip dysplasia a) pre-operative and b) at the latest follow-up. The right hip was operated on at 41 years of age with a follow-up of two years and the left at 40 years of age with a follow-up of three years. Both the centre-edge and acetabular angles are significantly improved in both hips.

Table I. The mean (SD, range; ${ }^{\circ}$ ) pre-operative and latest radiological and clinical parameters of the 26 hips

\begin{tabular}{llll}
\hline & Pre-operative (SD; range) & Latest follow-up (SD; range) & p value \\
\hline CE* angle of Wiberg $^{7}\left({ }^{\circ}\right)$ & $7.7(6.5 ;-10$ to 17$)$ & $27.0(6.6 ; 10$ to 36$)$ & $<0.001$ \\
Refined CE angle $^{12}\left({ }^{\circ}\right)$ & $-3.1(7.0 ;-18$ to 14$)$ & $13.0(6.5 ;-6$ to 25$)$ & $<0.001$ \\
Acetabular angle of Sharp $^{8}\left({ }^{\circ}\right)$ & $49.3(2.2 ; 45$ to 54$)$ & $38.9(4.0 ; 34$ to 52$)$ & $<0.001$ \\
Modified acetabular angle $^{13}\left({ }^{\circ}\right)$ & $53.2(2.7 ; 48$ to 58$)$ & $44.3(3.8 ; 38$ to 56$)$ & $<0.001$ \\
Lateralisation of the femoral head $^{15}(\mathrm{~mm})$ & $17.2(5.1 ; 9$ to 27$)$ & $15.9(4.6 ; 8$ to 26$)$ & 0.032 \\
Harris hip score & $74.9(9.3 ; 53$ to 86$)$ & $93.0(6.5 ; 77$ to 100$)$ & $<0.001$ \\
\hline
\end{tabular}

${ }^{*} \mathrm{CE}$, centre-edge

patients. An additional proximal femoral varus osteotomy was performed in one patient and a trochanteric advancement osteotomy in another at the same session. The mean follow-up period for the 26 hips was 3.3 years $\pm 1.6(2$ to 7.5).

There was a statistically significant difference between the mean values of all the pre-operative and latest radiological parameters (Table I; Figs 4 and 5). Improvement in the $\mathrm{CE}$ angle was seen in all hips and in the acetabular angle in all but one hip. The CE angle was considered to be pathological $\left(<20^{\circ}\right)$ in all hips pre-operatively, but in only two at the latest follow-up. The acetabular angle was also considered to be pathological $\left(>43^{\circ}\right)$ in all hips pre-operatively, and in only two at the latest follow-up. Lateralisation of the femoral head was reduced in 15 hips and unchanged in four. In the remaining seven hips, it increased.

Pre-operatively, seven hips had grade- 0 osteoarthritis, 18 grade 1 and one grade 2 according to Tönnis. ${ }^{9}$ At the latest follow-up all but one hip had the same pre-operative osteoarthritic grading. In this hip, which became infected, the grading decreased from 0 pre-operatively to 2 at 3.5 years after operation.

The difference between the pre-operative and latest mean values of the Harris hip scores was also statistically signifi-

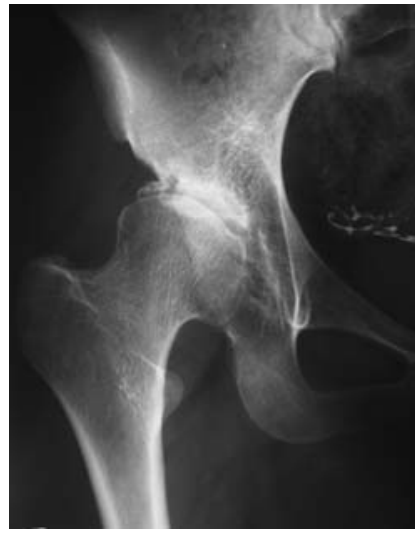

Fig. $5 a$

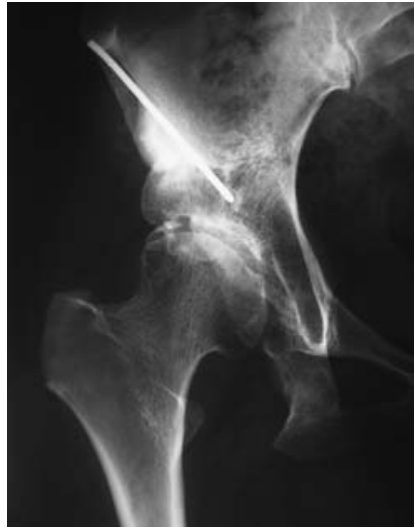

Fig. 5b
Anteroposterior radiographs of the right hip of a woman who was operated on at 34 years of age because of unilateral hip dysplasia a) pre-operative and b) at the latest follow-up. Two years after surgery there was significant improvement in the cover of the lateral femoral head and in the acetabular slope.

cant (Table I). The clinical scores of all the hips improved. Pre-operatively, eight hips were graded as good, 13 as fair and five as poor. At the latest assessment 18 hips were rated excellent, seven good and one fair. 
Complications. Deep-wound infection was seen in one patient at three months after operation. It resolved after surgical drainage and appropriate antibiotics. An intraoperative intra-articular fracture and asymptomatic nonunion of the ischial osteotomy were seen in one patient. However, the clinical assessment was considered to be excellent at 4.5 years after operation. Numbness in the distribution of the lateral femoral cutaneous nerve was noted in 11 patients but resolved completely in all within a year. Heterotopic ossification was not seen, and further surgery not performed in any of the hips.

\section{Discussion}

The most common useful site of correction for joint preservation in a congruous dysplastic hip is the periacetabular region where different types of pelvic osteotomy can be performed. ${ }^{17}$

To our knowledge, an incomplete iliac osteotomy has not been described for treating hip dysplasia in adolescents and adults. The acetabulum can be shifted anteriorly, laterally and inferiorly and it is possible to re-orientate and deepen it. A mean follow-up of nearly 3.5 years may be considered to be a shortcoming of our study, but we believe that the initial radiological and clinical results are significant.

There is a global primary deformity in acetabular dysplasia with poor anterior, lateral and posterior cover of the femoral head and not simply anterolateral acetabular maldirection, as is commonly assumed. ${ }^{18}$ The radiological results of our study showed that both the cover of the femoral head and slope of the acetabulum were significantly improved by this technique. However, when comparing the anterior and middle/posterior portions of the acetabulum the anterior correction seemed to be slightly better. This means that the osteotomy slightly retroverts the acetabulum while redirecting it. It is claimed that hips with a significant high or low degree of acetabular and femoral anteversion are unstable and thereby more prone to osteoarthritic changes. ${ }^{19}$ We do not have data comparing pre- and post-operative acetabular anteversion in our series, but we think that any decrease in acetabular anteversion due to the osteotomy is not significant since none of the patients had significant pain, clinical signs of hip instability or early radiological degenerative changes at the latest follow-up. When performing the osteotomy it appears that the more dysplastic the hip, the greater the risk of decreasing the acetabular anteversion due to the need for more acetabular redirection. We consider that this problem can be overcome by completing the iliac osteotomy towards the sciatic notch to control the acetabular anteversion in such hips, but have never undertaken such a modification.

Our technique reduced lateralisation of the femoral head in nearly two-thirds of the cases. The achievement of slight medial displacement of the centre of rotation of the hip may be an advantage.

Overcorrection after acetabular redirection may lead to impingement laterally, bringing the fovea into the weight- bearing area. ${ }^{20}$ In such hips the CE angle is $>40^{\circ}$. We did not see this and suggest that the technique provides controlled correction because of preservation of the posterior column. The clinical results are encouraging. Worsening of the osteoarthritic status was not encountered except in one hip.

The main advantage of this technique over other methods of triple pelvic osteotomy is the maintenance of an intact posterior column. This provides a stable osteotomy and allows minimal internal fixation and early post-operative mobilisation. ${ }^{21}$ Because the sciatic notch is not entered, damage to neurovascular structures is less likely. The iliac osteotomy is stable enough such that only one Kirschner wire is needed for fixation and early mobilisation, partial weight-bearing and active hip movements are strongly recommended, since perfect stability of the iliac osteotomy is present even without fixation in almost all cases. We plan to modify the technique by avoiding internal fixation and secondary operations for the removal of pins.

The dimensions of the true pelvis are not impaired if the posterior column is left intact, thus allowing unimpaired vaginal delivery. ${ }^{21}$ Our first patient with a follow-up of 7.5 years subsequently had three children, all of whom were delivered normally.

The main advantage of this technique compared with periacetabular ${ }^{22}$ and rotational acetabular osteotomies ${ }^{23}$ is its simplicity. The learning curve is not long, special instruments are not required, minimal blood transfusion is needed and there is no exposure to radiation.

There are four technical details which should be emphasised.

1) A small bone wedge is excised at the ischial osteotomy to facilitate acetabular redirection. This does not cause significant healing problems. Only one of our patients had nonunion of the ischial osteotomy.

2) The harvesting technique of the subcristal iliac bone graft avoids post-operative discomfort at the donor site and is cosmetic. No patient complained of the morbidity at the donor site.

3) The muscles and periosteum are not stripped from the medial aspect of the ilium and sartorius and rectus femoris are not detached. We believe that avoidance of unnecessary soft-tissue detachment may decrease intra-operative blood loss, avoid heterotopic ossification and facilitate post-operative rehabilitation.

4) The entire procedure can be performed with the patient supine and an adequate operative field for the ischial osteotomy can be obtained by placing a pillow under the buttock on the affected side.

Incomplete triple osteotomy is an effective technique for the treatment of residual hip dysplasia in adolescents and adults before advanced osteoarthritis develops. Although the initial radiological and clinical results seem to be satisfactory, long-term follow-up studies are needed.

No benefits in any form have been received or will be received from a commercial party related directly or indirectly to the subject of this article. 


\section{References}

1. Herring JA. Tachdjians's pediatric orthopaedics. Vol. 1. Third ed. Philadelphia: W. B. Saunders Co., 2002:587-96.

2. LeCoeur. Corrections des défaults d'orientation de l'articulation coxo-femorale par ostéotomie de l'isthme ilaque. Rev Chir Orthop Reparatrice Appar Mot 1965;51 211-12 (in French).

3. Carlioz H, Khouri N, Hulin P. Triple juxtacotyloid osteotomy. Rev Chir Orthop Reparatrice Appar Mot 1982;68:497-501 (in French).

4. Kotz R, Da Vid T, Helwig U, et al. Polygonal triple osteotomy of the pelvis: a correction for dysplastic hip joints. Int Orthop 1992;16:311-16.

5. Steel HH. Triple osteotomy of the innominate bone. J Bone Joint Surg [Am] 1973; 55-A:343-50.

6. Tönnis D, Behrens K, Tscharani F. A modified technique of the triple pelvic osteotomy: early results. J Pediatr Orthop 1981;1:241-9.

7. Wiberg G. Studies on dysplastic acetabula and congenital subluxation of the hip joint: with special reference to the complication of osteo-arthritis. Acta Chir Scand 1939;83(Suppl 58):1-135.

8. Sharp IK. Acetabular dysplasia: the acetabular angle. J Bone Joint Surg [Br] 1961; 43-B:268-72.

9. Tönnis D. Congenital dysplasia and dislocation of the hip in children and adults. Berlin, etc: Springer-Verlag, 1987:165-71.

10. Marafioti RL, Westin GW. Factors influencing the results of acetabuloplasty in children. J Bone Joint Surg [Am] 1980;62-A:765-9.

11. Kim HT, Kim JI, Yoo Cl. Diagnosing childhood dysplasia using the lateral margin of the sourcil. J Pediatr Orthop 2000;20:709-17.
12. Ogata S, Moriya H, Tsuchiya K, et al. Acetabular cover in congenital dislocation of the hip. J Bone Joint Surg [Br] 1990;72-B:190-6.

13. Agus H, Biçimoglu A, Ömeroglu H, Tümer Y. How should the acetabular angle of Sharp be measured on a pelvic radiograph? J Pediatr Orthop 2002;22:228-31.

14. Ömeroglu H, Biçimoglu A, Agus H, Tümer Y. Measurement of center-edge angle in developmental dysplasia of the hip: a comparison of two methods in patients under 20 years of age. Skeletal Radiol 2002;31:25-9.

15. Siebenrock KA, Leunig M, Ganz R. Periacetabular osteotomy: the Bernese experience. J Bone Joint Surg [Am]2001;83-A:449-55

16. Harris WH. Traumatic arthritis of the hip after dislocation and acetabular fractures: treatment by mold arthroplasty. J Bone Joint Surg [Am] 1969;51-A:737-55.

17. Millis MB, Kim YJ. Rationale of osteotomy and related procedures for hip preservation: a review. Clin Orthop 2002;405:108-21.

18. Murphy SB, Kijerwski PK, Millis MB, Harless A. Acetabular dysplasia in the adolescent and young adult. Clin Orthop 1990;261:214-23.

19. Tönnis D, Heinecke A. Acetabular and femoral anteversion: relationship with osteoarthritis of the hip. J Bone Joint Surg [Am] 1999;81-A:1747-70.

20. Søballe K. Pelvic osteotomy for acetabular dysplasia. Acta Orthop Scand 2003;74: 117-18.

21. Leunig M, Siebenrock KA, Ganz R. Rationale of periacetabular osteotomy and background work. J Bone Joint Surg [Am]2001;83-A:438-48.

22. Ganz R, Klaue K, Vinh TS, Mast JW. A new periacetabular osteotomy for the treatment of hip dysplasias: technique and preliminary results. Clin Orthop 1988;232:26-36.

23. Ninomiya S, Tagawa H. Rotational acetabular osteotomy for the dysplastic hip J Bone Joint Surg [Am] 1984;66-A:430-6. 